\title{
Seizure Onset Detection based on a Uni- or Multi-modal Intelligent Seizure Acquisition (UISA/MISA) System
}

Conradsen, Isa; Beniczky, Sándor; Wolf, Peter; Duun-Henriksen, Jonas; Sams, Thomas; Sørensen, Helge Bjarup Dissing

Published in:

2010 Annual International Conference of the IEEE Engineering in Medicine and Biology Society (EMBC)

Link to article, DOI:

10.1109/IEMBS.2010.5627218

Publication date:

2010

Document Version

Publisher's PDF, also known as Version of record

Link back to DTU Orbit

Citation (APA):

Conradsen, I., Beniczky, S., Wolf, P., Duun-Henriksen, J., Sams, T., \& Sørensen, H. B. D. (2010). Seizure Onset Detection based on a Uni- or Multi-modal Intelligent Seizure Acquisition (UISA/MISA) System. In 2010 Annual International Conference of the IEEE Engineering in Medicine and Biology Society (EMBC) (pp. 3269-3272). IEEE. I E E E Engineering in Medicine and Biology Society. Conference Proceedings https://doi.org/10.1109/IEMBS.2010.5627218

\section{General rights}

Copyright and moral rights for the publications made accessible in the public portal are retained by the authors and/or other copyright owners and it is a condition of accessing publications that users recognise and abide by the legal requirements associated with these rights.

- Users may download and print one copy of any publication from the public portal for the purpose of private study or research.

- You may not further distribute the material or use it for any profit-making activity or commercial gain

- You may freely distribute the URL identifying the publication in the public portal 


\title{
Seizure Onset Detection based on a Uni- or Multi-modal Intelligent Seizure Acquisition (UISA/MISA) System
}

\author{
Isa Conradsen $^{\dagger *}$, Sándor Beniczky* ${ }^{*}$ Peter Wolf*, Jonas Henriksen ${ }^{\dagger}$, Thomas Sams ${ }^{\dagger}$ and Helge B.D. Sorensen ${ }^{\dagger}$
}

\begin{abstract}
An automatic Uni- or Multi-modal Inteligent Seizure Acquisition (UISA/MISA) system is highly applicable for onset detection of epileptic seizures based on motion data. The modalities used are surface electromyography (sEMG), acceleration (ACC) and angular velocity (ANG). The new proposed automatic algorithm on motion data is extracting features as "log-sum" measures of discrete wavelet components. Classification into the two groups "seizure" versus "nonseizure" is made based on the support vector machine (SVM) algorithm.

The algorithm performs with a sensitivity of $91-100 \%$, a median latency of 1 second and a specificity of $100 \%$ on multi-modal data from five healthy subjects simulating seizures. The uni-modal algorithm based on sEMG data from the subjects and patients performs satisfactorily in some cases. As expected, our results clearly show superiority of the multimodal approach, as compared with the uni-modal one.
\end{abstract}

\section{INTRODUCTION}

More than 50 million people around the world suffer from epilepsy and about $25 \%$ of them cannot become seizure free. Patients dreading the next seizure onset has potential to become socialy isolated. Severe and sometimes fatal injuries can occur during seizures. An alarm system, capable of detecting seizures, could alert relatives and caretakers and ensure help for the patient. Several groups [1], [2] have already tried to develop such a system based on motion data, but none of them is performing well enough to reach clinical use. We therefore propose a new automatic detection algorithm capable of capturing the seizures with motor manifestations, without too many false alarms. It was decided in our previous study [3] to work further with movement sensors and surface electromyography (sEMG) registrations, as these provided promising results. Our new approach on these multi-modal motion data encompasses feature extraction with a discrete wavelet decomposition and an automatic classification with support vector machines (SVM). The MISA method including motion and sEMG data, was tested on 5 healthy subjects simulating seizures. However, due to impediments with the recruitment of patients, at present time it was only possible to test a uni-modal method on sEMG data alone, from 5 patients suffering from epilepsy.

Corresponding authors: Isa Conradsen, is conradsen@gmail.com, Helge BD Sorensen, hbs@elektro.dtu.dk

${ }^{\dagger}$ DTU Electrical Engineering, Ørsteds Plads, building 349, DK-2800 Kgs. Lyngby

*Danish Epilepsy Center, Kolonivej 1, DK-4293 Dianalund

\section{METHODOLOGY}

\section{A. Data Collection}

Data were recorded at the Danish Epilepsy Center in Dianalund, Denmark. The 5 healthy subjects were measured with 16 movement sensors, containing 3D accelerometers, 3D gyroscopes and 3D magnetometers, and 14 bipolar sEMG channels for 2-4 hours using our setup described in [3]. Each subject simulated 15 seizures in total, divided in the three types, myoclonic, tonic-clonic and versive. These are defined by epileptologists and described in details in [3]. The healthy volunteers carefully watched a video-recording with the movement pattern they had to imitate. An epileptologist explained them the typical aspects of the seizures, and the participants practised the movements before the recording. It was difficult to find patients with enough seizures with motor manifestations who could cooperate to wear all the equipment, so for this study the patients have only been measured with 4 sEMG channels with a sampling frequency of $1024 \mathrm{~Hz}$. The sEMG electrodes were placed on deltoid and tibialis anterior muscles on both sides of the body (active electrode on the muscle bulk, reference electrode on the tendon adjacent to it). The number and type of seizures along with the sex and age of the patients are listed in Table I. Furthermore the length of the signals for the testing phase of the classification is listed.

TABLE I

THE PATIENTS GENDER, AGE AND THE AMOUNT AND TYPE OF SEIZURES ALONG WITH THE LENGTH OF THE TEST FILE.

\begin{tabular}{l||c|r|r|l|r} 
& Gender & Age & $\begin{array}{r}\text { \# of } \\
\text { seizures }\end{array}$ & Seizure Type & $\begin{array}{r}\text { Length of } \\
\text { Test File [h] }\end{array}$ \\
\hline \hline Pt 1 & F & 2 & 13 & Tonic, Myoclonic & 12 \\
Pt 2 & F & 29 & 4 & Tonic-clonic & 27 \\
Pt 3 & M & 5 & 14 & Tonic, Spasm & 31 \\
Pt 4 & M & 48 & 10 & Tonic & 0.75 \\
Pt 5 & M & 30 & 11 & Tonic & 8 \\
\hline
\end{tabular}

\section{B. Data Processing}

The processing of data is split into three parts. The first part is the data partitioning, followed by the feature extraction and the last part is the classification into seizure and non-seizure events.

1) Data Partitioning: Data are split in smaller parts of seizure and non-seizure data to have more parts to choose from for the different iterations in the training and testing phases related to the classification. This provides a more 
reliable result. The data are partitioned based on which subject is measured: healthy subject or patient, respectively. For each subject several files are processed. A patient file containing seizures is divided in subparts as shown in Fig. 1, where each data part between the seizures is split into periods of 1 minute. This is long enough to ensure that the movements within the period make sense, and short enough to ensure that sufficiently many periods for training and testing are obtained. Between each period a sequence of 5 seconds is left unused to avoid correlation between two successive periods. In the files containing simulated seizures, the periods between these are left unused, since the healthy subjects were practicing for the seizure simulations in between seizure periods. A file without seizures is treated equally, regardless of whether it is from a healthy subject or a patient. The file is split into periods of 1 minute, with 5 seconds sequences left unused between each - just as explained above for the patient file containing seizures.

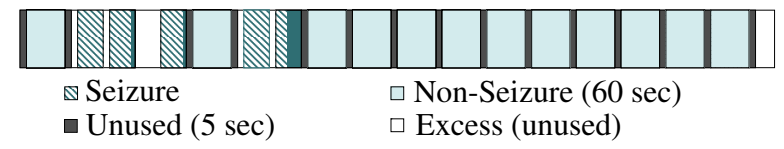

Fig. 1. The segmentation of a patient file containing five seizures. Between the seizures data are split into periods of 1 minute, with a sequence of 5 seconds left unused between successive periods.

2) Feature Extraction: Nijsen et al [1] showed that the wavelet decomposition would be a better choice for a feature than the short time Fourier transformation. The inherent properties of the wavelet transformation compared to the short time Fourier transformation gives a better timefrequency (time-scale) representation of normal movements versus seizures. As in [1] we have chosen to use the fifth Daubechies as the mother wavelet. The features are extracted from the discrete wavelet decomposition of windows of 1 second. The windows overlap by $50 \%$ and are filtered prior to the wavelet decomposition using a Hann filter of the same size as the window. This is done to smoothen the spectrum of the signal before processing it. The wavelet decomposition is performed through filtration with a high- and a low-pass filter as given by [4]:

$$
\begin{aligned}
A=v_{\text {low }}[m] & =\sum_{l=0}^{L-1} u[l] g[2 m-l] \\
D=v_{\text {high }}[m] & =\sum_{l=0}^{L-1} u[l] h[2 m+1-l]
\end{aligned}
$$

where 2 is the downsampling factor, $m$ and $l$ are the sample number in the signal, $L$ is the number of samples in the window and $g$ and $h$ are low- and high-pass filters, respectively. For each filtration the signal is then divided in an Approximation- (A) and a Detail- (D) signal. $y_{\text {low }}$ is the approximation signal, whereas $y_{\text {high }}$ is the detail signal. From each approximation signal a new step with filtrations is made by splitting as shown in Fig. 2. Each channel (ACC, ANG or EMG) is applied in the wavelet decomposition as $u(l)$.

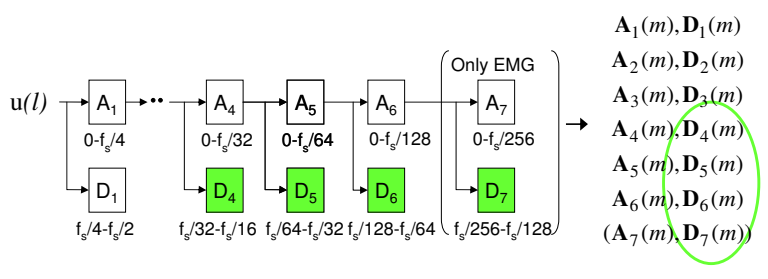

Fig. 2. The signal, $\mathrm{u}(l)$, is filtered and thereby split in approximation and detail signals, each approximation signal is further filtered. This is done six (seven) times. For the feature vector we only use the detail signals from the fourth to the sixth (seventh) sub-band $\left(f_{s}=1 \mathrm{kHz}\right)$. The frequencies beneath the squares state the bounds for the sub-band.

From a visual inspection based on a comparison of the spectral content for seizure and non-seizure events, respectively, the frequencies of interest were found to be in the lower range. Based on this conclusion, only the detail signals layer 4-6 (7) are further used for the feature extraction.

To decrease the amount of data entering the feature vector, a "log-sum" measure is calculated for each sub-band used.

$$
x_{j-3}=\log \left(\sum_{m=1}^{\frac{M}{2^{j}}}\left|D_{j}(m)\right|\right), \text { where } j=4,5,6(, 7)
$$

where $M$ is the number of samples in the signal $u(l), j$ is the sub-band number $(4,5,6(, 7))$ and $D(m)$ is the detail signal. For our data $M=120$ for ACC/ANG and $M=1024$ for EMG data. By applying the logarithm it is ensured that the smaller and more essential details are enhanced, while the larger and insignificant ones are reduced.

The feature vector, $\mathbf{x}$, is then collected as:

$$
\begin{aligned}
\mathbf{a} & =\left[x_{1, A C C_{1}}, \ldots, x_{3, A C C_{1}}, D_{1, A C C_{2}}, \ldots, x_{2, A C C_{69}}, x_{3, A C C_{69}}\right] \\
\mathbf{b} & =\left[x_{1, A N G_{1}}, \ldots, x_{3, A N G_{1}}, D_{1, A N G_{2}}, \ldots, x_{2, A N G_{69}}, x_{3, A N G_{69}}\right] \\
\mathbf{c} & =\left[x_{1, E M G_{1}}, \ldots, x_{4, E M G_{1}}, D_{1, E M G_{2}}, \ldots, x_{4, E M G_{14}}\right] \\
\mathbf{x}_{n} & =\left[\mathbf{a}_{n}, \mathbf{b}_{n}, \mathbf{c}_{n}\right]^{T}
\end{aligned}
$$

where $A C C_{1}$ means ACC channel 1 and so on and $n$ is the time index. For convenience the time index, $n$, is omitted in the previous equations.

For the ACC/ANG data, six steps of filtration are made, but only the detail signals from sub-band four to six are used further on. The sEMG data are filtered in seven steps and the detail signals from sub-band four to seven are used further on. These are also the sub-bands outlined in Fig. 2. These numbers of filtrations mean that we only use the frequencies 0.94-7.5 Hz for the ACC/ANG data and the frequencies from 4-64 Hz of the sEMG signals. The frequencies are chosen based on a visual inspection of the spectra of the signals.

The feature vector is now complete and can be submitted to a classifier.

3) Classification: The problem is to solve a binary classification problem with the classes Seizure and Non-seizure. The class Seizure contains all the seizures in the measurements, whereas the class Non-seizure contains everything else. This means that the class Non-seizure contains much 
more data than the class Seizure. As a classifier SVM is used, since it has proven to be better (than other complex algorithms such as artificial neural network) at handling data with very dissimilar amounts of data in the classes [5]. Data are divided into two groups, "train" and "test", whereas the classifier is trained on data from the "train" group. The data from the "test" group can then be classified with the classifier trained for the purpose. The classifier will return a positive or negative value for each " test" vector, dependent on whether it is estimated as belonging to a seizure period or not. The divisions into these groups of the healthy subject data are made randomly, for both seizure and non-seizure data, ensuring close to equal amounts of each data type in each group. For the patients, the first couple of seizures along with non-seizure data are used for training and the rest of the seizures with non-seizure data for testing. The division is made in this way to keep it causal and thereby imitate a real-time situation.

For the training, data is labeled:

$$
\left\{\mathbf{x}_{n}, y_{n}\right\}, n=1, \ldots, l, y_{n} \in\{-1,1\}, \mathbf{x}_{n} \in \mathfrak{R}^{d},
$$

where $l$ is the number of training examples, $\mathbf{x}_{n}$ is the feature vector ( $n$ is the time index) and $y_{n}$ the matching target, indicating which of the classes the feature vectors belong to, -1 for non-seizure and 1 for seizure.

A two-class linearly separable data set (where $d>2$ ) can be separated by a hyperplane described by:

$$
f\left(\mathbf{x}_{n}\right)=\mathbf{w} \cdot \mathbf{x}_{n}+b=0,
$$

where $\mathbf{w}$ is the normal to the hyperplane and $b$ is a shifting constant. The hyperplane is computed based on support vectors, which are the feature vectors that are placed closest to the hyperplane separating the two classes. These feature vectors from the two classes must satisfy:

$$
y_{n} \cdot\left(\mathbf{w} \cdot \mathbf{x}_{n}+b\right) \geq 1-\xi_{n}, \quad \text { where } \quad \xi_{n} \geq 0 \forall_{n},
$$

where $\xi_{n}$, a positive slack variable, is introduced to handle data, due that most classification problems are not completely separable. Data points assigned to the wrong side of the margin (defined by (7)) thereby have a penalty that increases with the distance to the margin.

To separate the two classes the problem of finding the optimal parameters, $\mathbf{w}$ and $b$, can be reduced to minimizing the performance function (8):

$$
\frac{1}{2}\|\mathbf{w}\|^{2}+C \sum_{n=1}^{l} \xi_{n} \quad \text { subject to } \quad y_{n} \cdot\left(\mathbf{w} \cdot \mathbf{x}_{n}+b\right) \geq 1-\xi_{n},
$$

where $C$ is a factor which sets the trade-off between the size of the margin and the penalty of the slack variable, $\xi_{n}$ [6].

For (8) to be minimized, each term should be minimized. Minimizing the first term means maximizing the margin between the support vectors of the two classes. The second term which encompass the slack variable is minimized by keeping the distance from incorrectly classified feature vectors to the margin as small as possible. When a feature vector is correctly classified $\xi_{n}$ is set to 0 , whereby the second term in (8) will be 0 . For a feature vector correctly classified, but placed on the wrong side of the margin, $\xi_{n}$ is between 0 and 1. Whereas it is above 1 , if the feature vector is wrongly classified. In the two latter cases the margin is attempted placed as close to these incorrectly classified feature vectors as possible to minimize the second term in (8).

To solve (8) Lagrange multipliers are multiplied and the equation is transformed from its primary form to the dual form, whereby it is possible to identify the parameters for the hyperplane which best separates the two classes. These steps are all performed in MATLAB by the SVM ${ }^{\text {light }}$ package specified in [7]. The package returns a classification-model based on the given training set, which can then be used to classify a test set.

\section{RESUlts AND Discussion}

The results are presented as sensitivity (the amount of the seizures that are detected), specificity (the amount of nonseizures that are not detected) and latency (the time it takes to detect the seizures after seizure onset). The specificity might not be the best measure for the number of false alarms, but for the healthy subjects the measurements were very compact and every movement was planned beforehand, so no other measure would provide a more reliable value. On the other hand, we have further chosen to calculate the false detection rate (FDR) for the epilepsy patients, which is the amount of false detections per hour. An optimal result has $100 \%$ sensitivity and specificity, a latency of 1 second (due to the window length) and an FDR of 0.

The results for the healthy subjects on multi-modal data are shown in Fig. 3. The mean sensitivity and specificity are calculated for 30 iterations, whereas for the latencies the median is provided. It is clearly seen that for subject $1-4$ the system has the highest sensitivity when all modalities are used. For the fifth subject the algorithm performs better with respect to sensitivity, if the EMG data is left out. Almost the same is seen concerning the specificity. The algorithm performs best for most subjects when all modalities are used, with exception of subject 2 where it is better if the EMG data is left out. With respect to the latency of the detections of the simulated seizures the result is also dependent on the subject, but most subjects have the best - or at least a very acceptable result when all modalities are used. There are a few outliers, but one should remember that it is the maximum latency that is depicted.

For the patients where only the sEMG data are provided, the results are shown in Fig. 4. This shows that the algorithm detects only half of the seizures for patient 1 . The reason is that the other half is myoclonus, which is very short lasting $(<0.5$ seconds $)$ and only happening in one muscle. This means that the muscles included in these seizures might not be the ones, which we have measured. It should be noticed that the seizures are detected at onset. Further it can be seen that there are only very few false positives $(0.08 / \mathrm{h})$. For patient 2 all seizures are detected, but most of them with a delay. The false positives are very few $(0.07 / \mathrm{h})$, which 


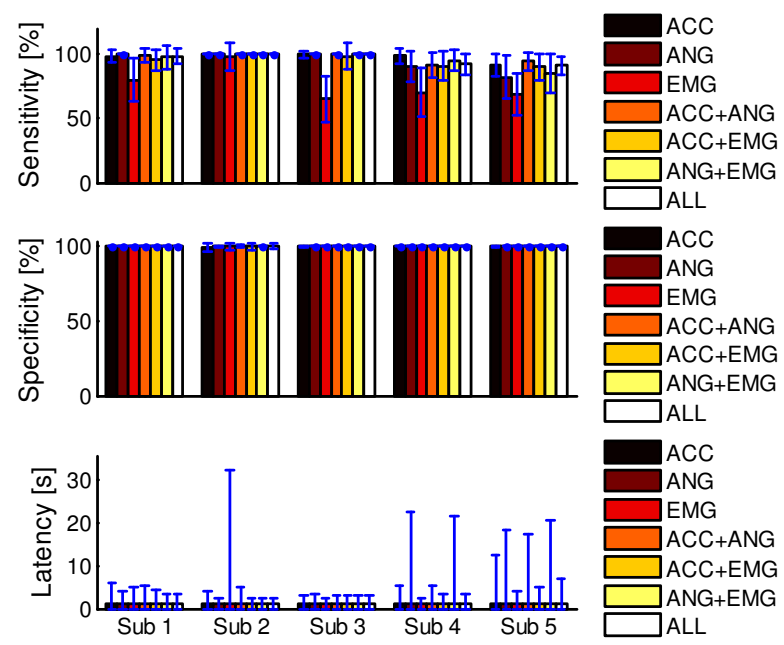

Fig. 3. The sensitivity, specificity and latency is showed for the results on the data from the five healthy subjects. For each subject the result is given for each modality alone and combinations of them. For the sensitivity and specificity the mean for 30 iterations (bars) and the standard deviation (blue lines) are shown. For the latency the median is shown by the bars (a bit difficult to see) and the blue line indicate maximum latency.

is important for an alarm system. For the third patient the algorithm is only able to detect one seizure, but neither does it capture any false positives. Notice that $50 \%$ of the seizures in the test data are spasms which the algorithm is not directly intended for. For the fourth patient all the seizures are detected at onset, but it has too many false alarms, the FDR might however be high due to the fact that we have less than an hour of data to test the algorithm on. For the last patient the algorithm is not able to detect all seizures, but those detected are however detected at onset. No postprocessing has yet been applied, which might lower the FDR for some patients. A change in the window size might be able to increase the sensitivity for patients with very short lasting seizures. Comparing the healthy subjects and the patients show equally well results using the UISA system on sEMG data. The better results on the healthy subjects using the MISA algorithm imply that better results might be achieved on patients using our multi-modal approach, which is the focus for our future experiments. The movements simulated by the healthy volunteers closely resembled those occurring during the seizures, therefore it is reasonable to assume that the signals recorded by the movement sensors are similar to what we would have recorded from patients with epilepsy. However the muscle-signals depend on the recruitment of the motor nerve cells. In the case of volunteers the motor cells are physiologically activated, while in the case of "real" seizures the recruitment is a pathological one. Thus we cannot exclude that the EMG signal recorded during the simulated seizures have different characteristics than the epileptic ones.

\section{CONCLUSION}

The automatic MISA system implemented is a new approach for motion data with feature extraction from discrete
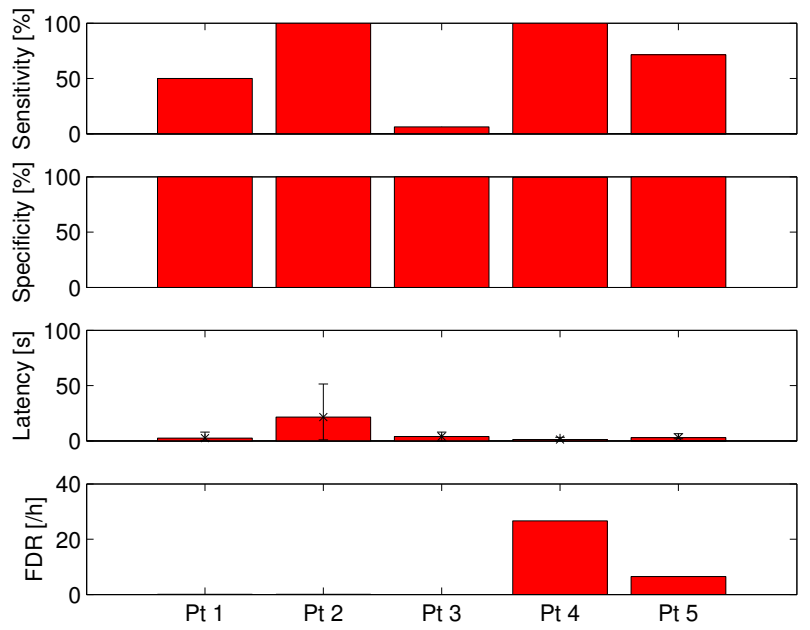

Fig. 4. The sensitivity, specificity, latency and false detection rate (FDR) are showed for the results on the EMG data from the five patients. The sensitivity, specificity and the FDR are shown as bars for each patient. For the latency the median is shown by the bars and the largest latency is indicated by the black line.

wavelet components. Data are classified with an SVM algorithm into the classes seizure and non-seizure. On the multimodal data from the healthy subjects the algorithm performs as intended, with a sensitivity of $91-100 \%$, a median latency of 1 second and a specificity of $100 \%$. Analysis of the sEMG data performed satisfactorily for both some of the patients and some of the healthy subjects imitating seizures. Our data on healthy subjects show the superiority of the multi-modal approach as compared to the unimodal one. At the moment, the device is a prototype for research use only. We have experienced that some patients feel uncomfortable wearing the suit containing the sensors. As a consequence suit and device setup is being modified for future experiments.

\section{REFERENCES}

[1] T. Nijsen, P. Cluitmans, P. Griep, and R. Aarts, "Short time Fourier and wavelet transform for accelerometric detection of myoclonic seizures," IEEE/EMBS Benelux, pp. 7-8, 2006.

[2] K. Cuppens, L. Lagae, B. Ceulemans, S. Van Huffel, and B. Vanrumste, "Detection of nocturnal frontal lobe seizures in pediatric patients by means of accelerometers: A first study.," in Conference proceedings:31st Annual International Conference of the IEEE Engineering in Medicine and Biology Society., vol. 1, pp. 6608-6611, 2009.

[3] I. Conradsen, S. Beniczky, P. Wolf, D. Terney, T. Sams, and H. Sorensen, "Multi-modal intelligent seizure acquisition (MISA) system-A new approach towards seizure detection based on full body motion measures.," in Conference proceedings:31st Annual International Conference of the IEEE Engineering in Medicine and Biology Society., vol. 1, pp. 2591-2595, 2009.

[4] J. Proakis and D. Manolakis, Digital signal processing: principles, algorithms, and applications. Macmillan Publishing Co., Inc. Indianapolis, IN, USA, 1992.

[5] C. Yang, J. Yang, and J. Wang, "Margin calibration in SVM classimbalanced learning," Neurocomputing, 2009.

[6] N. Cristianini and J. Shawe-Taylor, An introduction to support Vector Machines: and other kernel-based learning methods. Cambridge Univ Pr, 2000

[7] T. Joachims, "Learning to classify text using support vector machines: Methods, theory, and algorithms," Computational Linguistics, vol. 29, no. 4, pp. 656-664, 2002. 